ARTICLE OPEN

\title{
Changes in Parkinson's disease sleep symptoms and daytime somnolence after bilateral subthalamic deep brain stimulation in Parkinson's disease
}

\author{
Siddharth Kharkar ${ }^{1}$, Jonathan Richard Ellenbogen ${ }^{2}$, Michael Samuel ${ }^{3}$, Alexandra Rizos ${ }^{4}$, Monty Silverdale ${ }^{5}, \mathrm{~K}$ Ray Chaudhuri $^{3}$ and \\ Keyoumars Ashkan²
}

Introduction: Deep brain stimulation (DBS) markedly improves motor function in advanced Parkinson's disease (PD), but its effect on sleep is less clear. Patients and methods: Forty PD patients who had subthalamic DBS (STN-DBS) were identified from an ongoing non-motor naturalistic longitudinal study (NILS). All patients were followed up for at least 6 months, 26 patients had a 1 year follow-up. A total PDSS score of 100 or less, a score in any PDSS-item of 6 or less, and a Epworth score of 10 or more were classified as being significant. Results: Forty-five percent of patients reported significant improvement in the total PDSS score at 6 months, and $35 \%$ at 12 months. In terms of magnitude, the total PDSS score at 6 months was significantly improved from baseline while the improvement at 12 months was not statistically significant. The most frequently reported improvements were overall sleep quality and maintenance of sleep. Some patients reported worsening of the total PDSS score. More than half of the patients reporting daytime sleepiness at baseline had persistent sleepiness at 6 and 12 months. The mean Epworth Score did not improve because a significant number of patients without sleepiness at baseline reported new-onset sleepiness at 6 and 12 months. Neither medication changes nor motor improvement were consistently related to sleep changes after DBS. Conclusion: Subthalamic DBS is associated with a statistically and clinically significant, but variable, improvement in sleep as measured by the PDSS. The most frequent improvements were better overall sleep quality and better sleep maintenance.

npj Parkinson's Disease (2018)4:16; doi:10.1038/s41531-018-0053-5

\section{INTRODUCTION}

The high prevalence and marked impact of non-motor symptoms of Parkinson's disease (PD) on quality of life $\mathrm{e}^{1,2}$ are often underappreciated by treating physicians. ${ }^{3,4}$ Sleep problems are an integral aspect of the NMS of PD and are common in PD patients, and become increasingly more frequent and severe in advanced disease. ${ }^{5,6}$ Studies have reported that more than $95 \%$ of advanced PD patients have problems with sleep ${ }^{7}$ while recent studies in early PD have indicated an overall prevalence of about $49 \%{ }^{6}$ Practically any aspect of sleep may be affected, but the most consistently reported sleep problems in untreated PD are poor maintenance of sleep and sleep disturbed by nocturia.,

Dopaminergic therapy can improve aspects of sleep in PD patients. Long acting agents or short duration preparations given continuously (such as Apomorphine infusions) are particularly beneficial but even these strategies may not alleviate sleep symptoms completely. ${ }^{10-12}$ Conventional treatments for sleep problems, such as eszopiclone, ${ }^{13}$ clonazepam, $^{14}$ and doxepin ${ }^{15}$ may improve sleep in PD patients; but need to be used carefully since they have the potential to worsen daytime sleepiness, cognition and balance in PD patients.

Deep brain stimulation (DBS) is an attractive treatment option for patients where advanced therapies are indicated. ${ }^{16}$ While DBS markedly improves motor functioning ${ }^{17,18}$ its effect on sleep is less clear. There are only a handful of studies which utilize PD-specific sleep scales for evaluation of sleep changes after DBS, and some of these are compromised by small sample sizes and short duration of post-surgery follow-up.

We surveyed long term changes in subjective reporting of sleep symptoms (nocturnal and daytime) in 40 patients with Parkinson's disease who had Subthalamic Deep Brain Stimulation (STN-DBS), using the PDSS and Epworth Sleep Scale. We conducted this study with the aim of documenting changes in sleep symptoms after STN-DBS. A secondary aim was to explore the influence of motor improvement and medication changes on sleep symptoms after DBS.

\section{RESULTS}

Demographics and baseline characteristics

Patient demographics and baseline characteristics are presented in Table 1. A majority of patients (65\%) had significant overall impairment of sleep (total score $<=100$ ) at baseline. The most frequent sleep symptoms were bad overall sleep quality (PDSS-1: $75 \%$ ), difficulty maintaining sleep (PDSS-3: $82.5 \%$ ), and getting up to pass urine (PDSS-8: $80 \%$ ). $40 \%$ (16/40) patients had significant

\footnotetext{
${ }^{1}$ Department of Neurology, Wockhardt Hospitals, Mumbai, Maharashtra, India; ${ }^{2}$ Department of Neurosurgery, King's College Hospital NHS Foundation Trust, London, UK; ${ }^{3}$ Department of Neurology, King's College Hospital NHS Foundation Trust, London, UK; ${ }^{4}$ EUROPAR Offices, King's College Hospital NHS Foundation Trust, London, UK and ${ }^{5}$ Department of Neurology, Salford Royal NHS Foundation Trust, Manchester, UK

Correspondence: Jonathan Richard Ellenbogen (jellenbogen@nhs.net)

These authors contributed equally: K Ray Chaudhuri, Keyoumars Ashkan.
}

Received: 10 October 2017 Revised: 16 April 2018 Accepted: 2 May 2018

Published online: 25 May 2018 
daytime sleepiness at baseline as measured by the Epworth sleepiness scale.

\section{Sleep changes after DBS}

Forty-five percent (18/40) patients displayed significant improvement in the total PDSS score at 6 months, and 35\% (9/26) at 12 months. In terms of magnitude, the total PDSS score improved at 6 months by $23.5 \%$ (from a median score of 91.5 to $113.5, p=$ 0.001 ). The median score at 12 months (median $=102$ ) was higher than that at baseline, but this difference was not statistically significant $(p=0.07)$.

Table 1. Demographics

Mean (SD) or number (percentage)

Number of patients

Baseline

6 month follow-up

1 year follow-up

40

Gender (Females)

40

26

Age at onset of symptoms

$1537.5 \%)$

Age at the time of DBS surgery

$48.7 \pm 7.7$

Duration of symptoms before DBS

surgery (years)

PDSS-total $(<=100)$

$59.6 \pm 8.3$

$10.7 \pm 4.5$

PDSS-1: Overall sleep quality

$26(65 \%)$

PDSS-2: Difficulty falling asleep

$30(75 \%)$

$15(37.5 \%)$

$33(82.5 \%)$

PDSS-4: Restlessness of legs or arms

$18(45 \%)$

PDSS-5: Fidgeting in bed

$19(47.5 \%)$

PDSS-6: Distressing dreams

$9(22.5 \%)$

PDSS-7: Distressing hallucinations at night

PDSS-8: Getting up to pass urine

$1(2.5 \%)$

$32(80 \%)$

$4(10 \%)$

immobility

PDSS-10: Numbness or tingling of

$10(25 \%)$ arms/legs

PDSS-11: Muscle cramps while sleeping 18 (45\%)

PDSS-12: Painful posturing on waking $17(42.5 \%)$

PDSS-13: Tremor on waking 21 (52.5\%)

PDSS-14: Tired and sleepy on waking 23 (57.5\%)

PDSS-15: Unexpectedly falling asleep 17 (42.5\%)

during the day

Epworth sleepiness scale

$16(40 \%)$
The most frequently reported changes (Fig. 1) were improved overall sleep quality (PDSS-1: $55 \%$ and $46 \%$ of patients at 6 months and 12 months, respectively) and improved maintenance of sleep (PDSS-3: $62.5 \%$ and $62 \%$ of patients at 6 and 12 months). Patients also frequently reported improvement in tremor on waking (PDSS13: $40 \%$ and $27 \%$ of patients at 6 and 12 months) and painful posturing of legs in the morning (PDSS-12: $30 \%$ and $38 \%$ of patients at 6 and 12 months). At 6 months, the median improvements in all of these aspects of sleep were statistically significant (Fig. 2). 1 year improvement showed a similar pattern, but only the improvements in overall sleep quality, sleep maintenance, and tremor on waking were statistically significant, although this may be because of small numbers who reached 1 year follow up.

$7.5 \%(3 / 40)$ patients reported significant worsening of the total PDSS score at 6 months, and 19\% (5/26) at 12 months. In addition, for each sleep symptom, some patients reported deterioration (Fig. 1). In particular, patients reported a very variable response in terms of feeling tired and sleepy after waking up (PDSS-14). Patients frequently reported feeling less tired and sleepy after waking (47 and $35 \%$ at 6 months and 12 months) but, a substantial number of other patients also reported being more tired and sleepy on awakening (22.5\% at 6 months and $35 \%$ at 12 months) and hence there was no improvement in the PDSS-14 median score.

Of the 16 patients with significant daytime sleepiness at baseline as measured by the ESS, 56\% (9/16) had persistent daytime sleepiness at 6 months and $81 \%(9 / 11)$ at 12 months. In terms of magnitude, the Epworth Score did not improve because a significant number of patients without sleepiness at baseline reported new-onset sleepiness at 6 months $(33 \%, 8 / 24)$ and at 12 months $(20 \%, 3 / 15)$.

There was no statistically significant difference in the percentage improvement in the PDSS total scores between patients who showed moderate improvement of UPDRS scores after levodopa challenge, as compared to those who showed marked improvement.

\section{Changes in medications after DBS}

The mean LED dose decreased by $25 \%$ at 6 months (from $1051 \pm$ $413 \mathrm{mg}$ at baseline to $786 \pm 340 \mathrm{mg}$ ) and by $30.5 \%$ (to $730 \pm$ $417 \mathrm{mg}$ ) at the 1 year follow-up. We did not detect an association between decrease of dopaminergic medications and changes in the total PDSS score. At 6 months, larger decreases in total LEDD were weakly associated with a deterioration in the Epworth Sleepiness score (Rho: $-0.31, p=0.049$ ). No such relationship was found at 12 months.

\section{Changes in motor scores after DBS}

As expected, the SCOPA-motor score improved in most patients. The mean SCOPA-motor score improved by $35 \%$ at 6 months (from $25.3 \pm 10.1$ to $16.4 \pm 8.8, p<0.0001$ ) and by $40 \%$ at

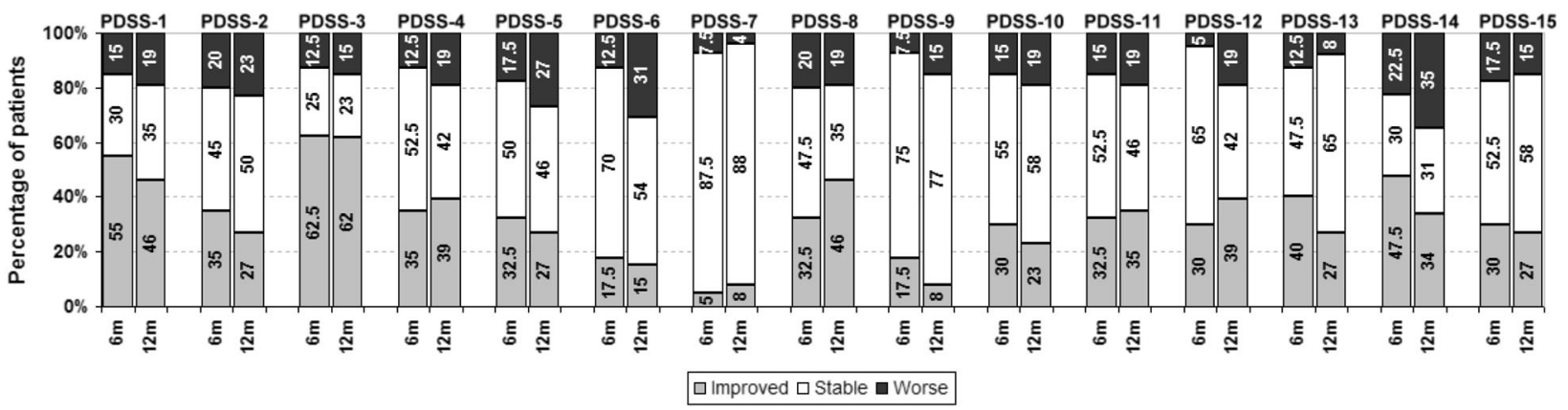

Fig. 1 Percentage of patients reporting a change in PDSS sub-scores at 6 and 12 months 


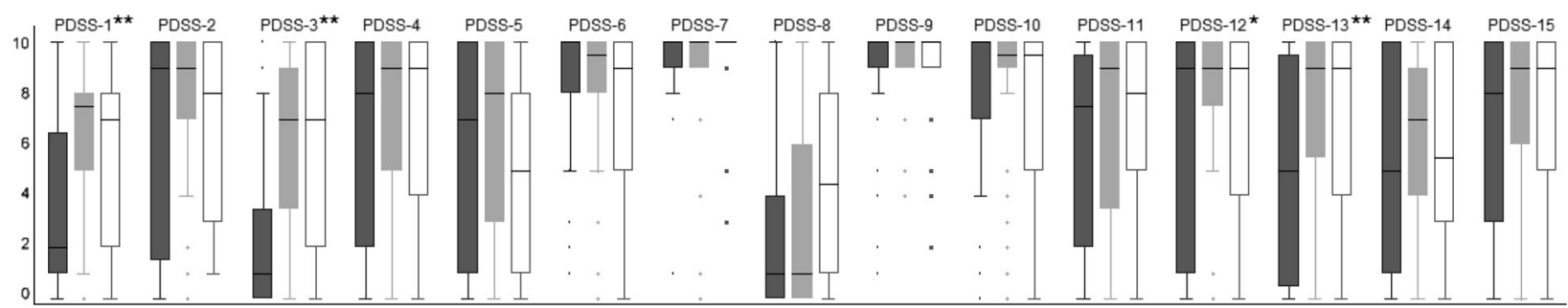

Fig. 2 Median and range of PDSS sub-scores at baseline, 6 and 12 months. (*6 month score statistically different from baseline, **both 6 and 12 month scores statistically different from baseline)

12 months (to $15.2 \pm 8.15, p<0.0001$ ). No clear relationship was discernible between improvement in SCOPA-motor scores and global improvement of sleep (total PDSS score) at either 6 or 12 months (Spearman's correlation).

\section{DISCUSSION}

In this study, subthalamic DBS was followed by a statistically and clinically significant, but variable, improvement in sleep as measured by the PDSS. The most frequent improvements were better overall sleep quality and better sleep maintenance. However, a variable but significant proportion of patients experienced deterioration in different aspects of sleep (Fig. 1). Sleep improvement after DBS did not correlate with improvement in UPDRS scores after levodopa challenge testing prior to DBS insertion, medication changes after DBS or motor improvement after DBS. At this point, we believe that it is reasonable to counsel patients that while different aspects of their sleep may improve or deteriorate after DBS; the most likely possibility is that they will have an overall improvement in their sleep.

The present study is the largest study utilizing a PD-specific sleep symptom questionnaire. A previous large study of sleep symptoms in PD after unilateral STN-DBS did not utilize a PDspecific sleep questionnaire, although the results reported were comparable to the present study. ${ }^{19}$ Polysomnography (PSG) studies of PD patients after DBS have documented a reduction in sleep disturbance, decrease in wakefulness after sleep onset, improvement in sleep efficiency and total sleep time, and increased REM sleep. ${ }^{20-23}$ These PSG changes are compatible with the changes documented in our study.

There is considerable variation in the results of previous studies utilizing the PDSS on post-DBS sleep changes ${ }^{24-27}$ (Table 2). The most consistent and significant change is seen in sleep maintenance (PDSS-3), in keeping with our study. It is possible that patients interpret this change as an improvement in their overall sleep quality (PDSS-1). In addition, we also noted substantial improvement in tremor and painful posturing after waking (PDSS-12 and 13), which is likely related to better control of motor symptoms by DBS even before the morning dose of dopaminergic medication is ingested.

Previous research has suggested that a marked decrease in dopaminergic medications (by $79 \%$ or more) after STN-DBS may worsen or lead to the emergence of restless legs syndrome and REM behavior disorder. ${ }^{28,29}$ In this study, a significant number of patients developed worsening restlessness of legs, distressing dreams and hallucinations at night (Fig. 2: PDSS-4, PDSS-6, and PDSS-7) even though the decrease in dopaminergic medications was modest. There is uncertainty regarding the relationship between leg motor restlessness in PD and true $\mathrm{RLS}^{30,31}$ and polysomnography is required for the definitive diagnosis of RBD. ${ }^{32}$ Hence, these findings should be interpreted with caution.

The PDSS assesses nocturnal problems, sleep disturbances and excessive daytime sleepiness and is composed of 15 items, addressing nocturnal symptoms commonly associated with PD (insomnia, nocturia, nocturnal motor symptoms, etc.). Each item is rated on a visual analog scale (VAS) from 0 (severe or always present) to 10 (never or not present). The total score is obtained by summing the items. The time frame is the previous week. It is specific for PD, is extensively used and validated and has been shown to be responsive to changes and is recommended by the MDS Task Force. ${ }^{33}$ Studies have also suggested data from PDSS correlate with PSG based datasets and worldwide reports from studies using PDSS indicate that scores below PDSS-total of 100 indicate abnormal sleep. The PDSS is useful to identify PD patients who will have abnormal PSG recordings, ${ }^{34}$ but the correlation between subjective and objective measures of sleep can be imperfect. ${ }^{35}$ We believe that subjective and objective measures of sleep are complimentary; neither one is necessarily "superior" to the other. Although it is clear that subjective sleep assessments are fundamental to complaints of insomnia and non-restorative sleep, two conditions associated with considerable morbidity and impairment in PD. ${ }^{36}$

Arnulf et al. ${ }^{20}$ found that night-time awakenings, a normal phenomenon, were often followed by dystonia and extended awakenings. They hypothesized that the continuous nature of DBS stimulation may provide better motor control at night, preventing such extended awakenings. Our univariate analyses indicated that improvement in motor function was not sufficient for an improvement in sleep maintenance; however its contribution needs to be evaluated with multivariate analysis in a larger sample. We detected an increase in daytime sleepiness associated with reduction of high doses of total LEDD. This is possibly the result of falling in the trough of the sleep-response to levodopa, wherein more moderate doses of levodopa are associated with sleepiness and higher doses with better wakefulness, as described by Bliwise et al. ${ }^{37}$

In addition to motor improvement and medication changes, multiple other factors may affect post-DBS sleep, including changes in depressive symptoms ${ }^{38}$ and possibly a direct effect of DBS on sleep structures. It is a difficult task to assess the relative contributions of these factors. GPi-DBS is usually followed by smaller decrease in dopaminergic medication ${ }^{39}$ and it may be possible to gain a better understanding of the relative importance of motor improvement without this additional confounder. In a randomized trial of GPi-DBS versus STN-DBS, ${ }^{39}$ the magnitude of sleep improvement measured by PDSS was similar in both groups, possibly indicating a durable effect common to both GPi and STN DBS that surpasses any effect of dopaminergic medication withdrawal.

There are limitations to our study. First, we did not assess sleep architecture formally, as discussed above. However, the globally validated PDSS use does offset the lack of PSG and additionally allows inclusion of motor and other PD symptoms which affect sleep. Second, we did not assess the effect of depression. Changes in mood which may occur in some patients after STN-DBS ${ }^{38}$ could have worsened sleep symptoms ${ }^{40}$ and diluted our results. Third, we did not undertake multivariate analysis. In our view, multivariate analyses could be misleading given the relatively small patient population. Since this was a preliminary study, we did not use a multiple comparison correction such as Bonferroni, which 


nPj Changes in Parkinson's disease sleep symptoms and daytime
S Kharkar et al.

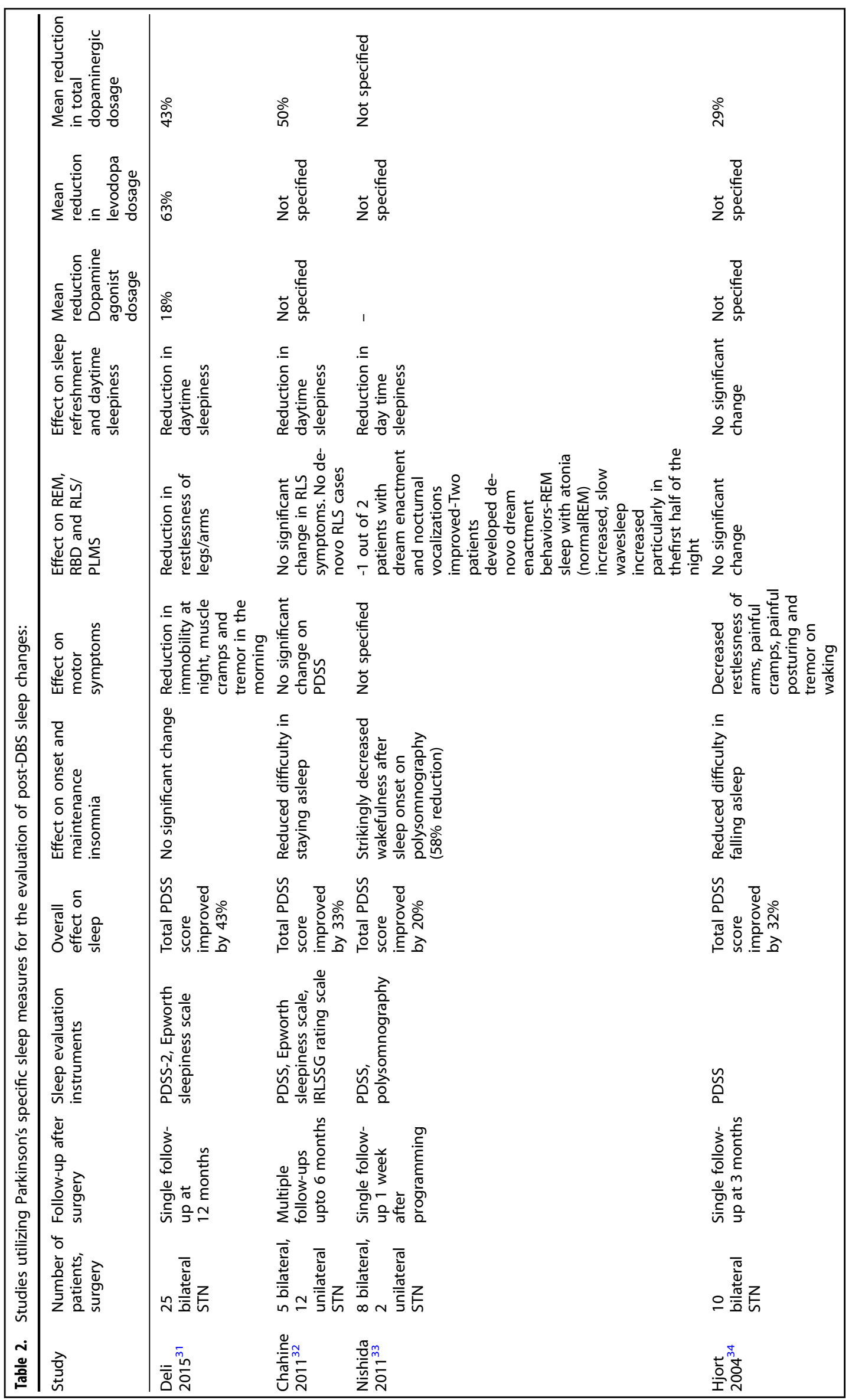


can substantially increase the chance of a type II error. ${ }^{41}$ Finally, the cut-offs we used for defining significant change in sleep symptoms need to be prospectively validated.

In conclusion, our study confirms improvement in some sleep symptoms after STN-DBS, in particular overall sleep quality and sleep maintenance. Our data indicates that improvement in motor function is not sufficient for sleep improvement. Further studies need to focus on the reasons for these improvements with the goal of refining DBS and medication management strategies after STN-DBS to maximize sleep improvement.

\section{METHODS}

Patients

40 patients who had STN-DBS for Parkinson's disease and were enrolled in the on-going non-motor naturalistic longitudinal study (NILS, UK clinical research network number 10084). The NILS study received ethical approval from all local Research Ethics Committees at each centre, and all patients provided informed written consent for inclusion in the study. All patients were followed up for at least 6 months, 26 of these patients also had a 1 year follow-up.

All patients met British Brain Bank criteria ${ }^{42}$ for PD, and were selected for DBS due to insufficient control of their motor symptoms by medication. Pre-surgery response to levodopa was verified in all cases by a $>30 \%$ improvement in the Unified Parkinson's Disease Rating Scale (UPDRS)-III motor score after a standard levodopa challenge dose. None of the patients had significant psychiatric illnesses or dementia which would preclude DBS implantation. All patients had pre-operative full multidisciplinary assessment by our dedicated DBS team, which includes a neuropsychologist and a neuropsychiatrist.

All patients in this study had bilateral subthalamic implantation of DBS electrodes, followed by initiation of stimulation within 6 weeks. DBS programmer settings were changed per clinical requirements at the discretion of the treating physician / DBS specialist nurse. Patients were evaluated at fixed intervals per the NILS protocol: within one month before DBS surgery, 6 months after surgery, and yearly thereafter. All 40 patients had a baseline and 6 month evaluation. At the present time, 26 patients have had the 12 month evaluation.

\section{Measurement instruments}

1. Sleep symptoms were assessed by the Parkinson's Disease Sleep Scale (PDSS), ${ }^{36}$ a patient reported 15 item scale with each item weighed from 0 to 10. Lower scores on each item represent worse symptoms: 0 indicates that the patient "always" has the symptom and 10 represents an answer of "never". Correspondingly, the total PDSS score varies between 0 (worst) and 150 (best).

2. Daytime sleepiness was assessed using the Epworth Sleepiness Scale, $^{43}$ which is an 8 -item scale that assesses the likelihood of falling asleep while performing common tasks during the day. A score of 0 indicates no daytime sleepiness, while the maximum score of 24 indicates the highest level of daytime sleepiness in all tested situations.

3. Motor improvement was measured by the motor subpart of the SCOPA scale (SCOPA-motor) ${ }_{14}^{44}$ a 21 item scale which correlates strongly with the corresponding subpart of UPDRS-III. ${ }^{44,45}$ Higher scores on SCOPA-motor represent worse motor symptoms, the maximum score is 75 .

\section{Categorization of scores}

Patients with a total PDSS score of 100 or less, ${ }^{10}$ a score in any PDSS-item of 6 or less, and a Epworth score of 10 or more ${ }^{46}$ were classified as having significant problems. A change of the total PDSS score by 20 points or more, or in one of the sub-scores by 2 points or more was considered clinically significant. Follow-up scores were classified as improved, stable or worse as compared to the baseline scores. The criteria for defining clinically significant change in PDSS scores were based on our clinical experience and have not been formally validated. The percentage improvement in UPDRS score after levodopa challenge was categorized into two categories: "Moderate" if the improvement was between $30-65 \%$ and "Marked" if the improvement was $65 \%$ or above.
Medication changes

The levodopa equivalent daily dose (LEDD) was computed according to the widely accepted method described by Tomlinson et al. ${ }^{47}$

\section{Statistical analysis}

Data analysis was done in Stata 12 (Stata Corp, Texas). Normality was tested using the Shapiro-Wilk test. For pre-DBS and post-DBS comparisons, the Wilcoxon signed-rank test was used for comparing non-normally distributed variables and the paired student $\mathrm{t}$-test was used for normally distributed variables, corresponding non-paired tests were used for other comparisons.

Data availability statement

The data is maintained by Ms. Alexandra Rizos, Research Manager at Kings College Hospital, and can be made available for review upon request.

\section{ACKNOWLEDGEMENTS}

We would like to thank Dr. Dhaval Trivedi for his support during preparation of this manuscript.

\section{AUTHOR CONTRIBUTIONS}

Drs. S.K., M.S., K.A., M.S., A.R., and K.R.C. designed and conducted the study, including patient recruitment, data collection, and data analysis. Dr. S.K. prepared the manuscript draft in collaboration with Drs. J.R.E, M.S., K.A., and K.R.C. All authors approved the final manuscript. All authors had complete access to the study data.

\section{ADDITIONAL INFORMATION}

Competing interests: The authors have received educational grants from deep brain stimulation manufacturers independent of this study.

Publisher's note: Springer Nature remains neutral with regard to jurisdictional claims in published maps and institutional affiliations.

\section{REFERENCES}

1. Chaudhuri, K. R. et al. The metric properties of a novel non-motor symptoms scale for Parkinson's disease: results from an international pilot study. Mov. Disord. 22, 1901-1911 (2007).

2. Barone, P. et al. The PRIAMO study: a multicenter assessment of nonmotor symptoms and their impact onquality of life in Parkinson's disease. Mov Disord. 24, 1641-1649 (2009).

3. Todorova, A., Jenner, P. \& Ray Chaudhuri, K. Non-motor Parkinson's: integral to motor Parkinson's, yet often neglected. Pract. Neurol. 14, 1-13 (2014).

4. Shulman, L. M., Taback, R. L., Rabinstein, A. A. \& Weiner, W. J. Non-recognition of depression and other non-motor symptoms in Parkinson's disease. Park. Relat. Disord. 8, 193-197 (2002).

5. Diederich, N. J., \& McIntyre, D. J. Sleep disorders in Parkinson's disease: many causes, few therapeutic options. J Neurol Sci. 314, 12-19 (2012).

6. Riedel, O. et al. Frequency of dementia, depression, and other neuropsychiatric symptoms in 1,449 outpatients with Parkinson's disease. J. Neurol. 257, 1073-1082 (2010).

7. Lees, A. J., Blackburn, N. A. \& Campbell, V. L. The nighttime problems of Parkinson's disease. Clin. Neuropharmacol. 11, 512-519 (1988).

8. Bušková, J. et al. Sleep disturbances in untreated Parkinson's disease. J. Neurol. 258, 2254-2259 (2011).

9. Ferreira, T., Prabhakar, S. \& Kharbanda, P. S. Sleep disturbances in drug naïve Parkinson's disease (PD) patients and effect of levodopa on sleep. Ann. Indian Acad. Neurol. 17, 416-419 (2014).

10. Ray Chaudhuri, K. et al. Improvements in nocturnal symptoms with ropinirole prolonged release in patients with advanced Parkinson's disease. Eur. J. Neurol. 19, 105-113 (2012).

11. Trenkwalder, C. et al. Rotigotine effects on early morning motor function and sleep in Parkinson's disease: a double-blind, randomized, placebo-controlled study (RECOVER). Mov. Disord. 26, 90-99 (2011).

12. Dusek, P. et al. Effects of ropinirole prolonged-release on sleep disturbances and daytime sleepiness in Parkinson disease. Clin. Neuropharmacol. 33, 186-190 (2010).

13. Menza, M. et al. Treatment of insomnia in Parkinson's disease: a controlled trial of eszopiclone and placebo. Mov. Disord. 25, 1708-1714 (2010). 
14. Olson, E. J., Boeve, B. F. \& Silber, M. H. Rapid eye movement sleep behaviour disorder: demographic, clinical and laboratory findings in 93 cases. Brain 123(Pt 2), 331-339 (2000).

15. Rios Romenets, S. et al. Doxepin and cognitive behavioural therapy for insomnia in patients with Parkinson's disease--a randomized study. Park. Relat. Disord. 19, 670-675 (2013).

16. Odin, P. et al. Collective physician perspectives on non-oral medication approaches for the management of clinically relevant unresolved issues in Parkinson's disease: Consensus from an international survey and discussion program. Park. Relat. Disord. 21, 1133-1144 (2015).

17. Weaver, F. M. et al. Bilateral deep brain stimulation vs best medical therapy for patients with advanced Parkinson disease: a randomized controlled trial. JAMA 301, 63-73 (2009).

18. Deuschl, G. et al. A Randomized Trial of Deep-Brain Stimulation for Parkinson's Disease. N. Engl. J. Med. 355, 896-908 (2006).

19. Amara, A. W. et al. Unilateral subthalamic nucleus deep brain stimulation improves sleep quality in Parkinson's disease. Park. Relat. Disord. 18, 63-68 (2012).

20. Arnulf, I. et al. Improvement of sleep architecture in PD with subthalamic nucleus stimulation. Neurology 55, 1732-1734 (2000).

21. Monaca, C. et al. Effects of bilateral subthalamic stimulation on sleep in Parkinson's disease. J. Neurol. 251, 214-218 (2004).

22. Cicolin, A. et al. Effects of deep brain stimulation of the subthalamic nucleus on sleep architecture in parkinsonian patients. Sleep. Med. 5, 207-210 (2004).

23. Iranzo, A., Valldeoriola, F., Santamaría, J., Tolosa, E. \& Rumià, J. Sleep symptoms and polysomnographic architecture in advanced Parkinson's disease after chronic bilateral subthalamic stimulation. J. Neurol. Neurosurg. Psychiatry 72, 661-664 (2002).

24. Deli, G. et al. Bilateral Subthalamic Stimulation can Improve Sleep Quality in Parkinson's Disease. J. Park. Dis. 5, 361-368 (2015).

25. Chahine, L. M., Ahmed, A. \& Sun, Z. Effects of STN DBS for Parkinson's disease on restless legs syndrome and other sleep-related measures. Park Relat. Disord. 17, 208-211 (2011)

26. Nishida, N. et al. Subthalamic nucleus deep brain stimulation restores normal rapid eye movement sleep in Parkinson's disease. Mov. Disord. 26, 2418-2422 (2011).

27. Hjort, N., Østergaard, K. \& Dupont, E. Improvement of sleep quality in patients with advanced Parkinson's disease treated with deep brain stimulation of the subthalamic nucleus. Mov. Disord. 19, 196-199 (2004).

28. Kim, Y. E. et al. Rapid eye movement sleep behavior disorder after bilateral subthalamic stimulation in Parkinson's disease. J. Clin. Neurosci. 22, 315-319 (2015).

29. Kedia, S., Moro, E., Tagliati, M., Lang, A. E. \& Kumar, R. Emergence of restless legs syndrome during subthalamic stimulation for Parkinson disease. Neurology 63, 2410-2412 (2004).

30. Suzuki, K. et al. Nocturnal disturbances and restlessness in Parkinson's disease: using the Japanese version of the Parkinson's disease sleep scale-2. J. Neurol. Sci. 318, 76-81 (2012).

31. Gjerstad, M. D., Tysnes, O. B. \& Larsen, J. P. Increased risk of leg motor restlessness but not RLS in early Parkinson disease. Neurology 77, 1941-1946 (2011).

32. Boeve, B. F. REM sleep behavior disorder: Updated review of the core features, the REM sleep behavior disorder-neurodegenerative disease association, evolving concepts, controversies, and future directions. Ann. N.Y. Acad. Sci. 1184, 15-54 (2010).
33. Högl, B. et al. Scales to assess sleep impairment in Parkinson's disease: critique and recommendations. Mov. Disord. 25, 2704-2716 (2010).

34. Dhawan, V. et al. The range and nature of sleep dysfunction in untreated Parkinson's disease (PD). A comparative controlled clinical study using the Parkinson's disease sleep scale and selective polysomnography. J. Neurol. Sci. 248, 158-162 (2006).

35. Kaplan, K. A. et al. When a gold standard isn't so golden: Lack of prediction of subjective sleep quality from sleep polysomnography. Biol. Psychol. 123, 37-46 (2017).

36. Chaudhuri, K. R. et al. The Parkinson's disease sleep scale: a new instrument for assessing sleep and nocturnal disability in Parkinson's disease. J. Neurol. Neurosurg. Psychiatry 73, 629-635 (2002).

37. Bliwise, D. L. et al. Daytime alertness in Parkinson's disease: potentially dosedependent, divergent effects by drug class. Mov. Disord. 27, 1118-1124 (2012).

38. Strutt, A. M., Simpson, R., Jankovic, J. \& York, M. K. Changes in cognitive-emotional and physiological symptoms of depression following STN-DBS for the treatment of Parkinson's disease. Eur. J. Neurol. 19, 121-127 (2012).

39. Odekerken, V. J. J. et al. Subthalamic nucleus versus globus pallidus bilateral deep brain stimulation for advanced Parkinson's disease (NSTAPS study): a randomised controlled trial. Lancet Neurol. 12, 37-44 (2013).

40. Murphy, M. J. \& Peterson, M. J. Sleep disturbances in depression. Sleep. Med. Clin. 10, 17-23 (2015).

41. Perneger, T. V. What's wrong with Bonferroni adjustments. BMJ 316, 1236-1238 (1998).

42. Hughes, A. J., Daniel, S. E., Kilford, L. \& Lees, A. J. Accuracy of clinical diagnosis of idiopathic Parkinson's disease: a clinico-pathological study of 100 cases. J. Neurol. Neurosurg. Psychiatry 55, 181-184 (1992).

43. Johns, M. W. A new method for measuring daytime sleepiness: the Epworth sleepiness scale. Sleep 14, 540-545 (1991).

44. Marinus, J. et al. A short scale for the assessment of motor impairments and disabilities in Parkinson's disease: the SPES/SCOPA. J. Neurol. Neurosurg. Psychiatry 75, 388-395 (2004).

45. Martínez-Martín, P. et al. The SCOPA-Motor Scale for assessment of Parkinson's disease is a consistent and valid measure. J. Clin. Epidemiol. 58, 674-679 (2005).

46. Johns, M. W. Daytime sleepiness, snoring, and obstructive sleep apnea. The Epworth Sleepiness Scale. CHEST J. 103, 30 (1993).

47. Tomlinson, C. L. et al. Systematic review of levodopa dose equivalency reporting in Parkinson's disease. Mov. Disord. 25, 2649-2653 (2010).

Open Access This article is licensed under a Creative Commons Attribution 4.0 International License, which permits use, sharing, adaptation, distribution and reproduction in any medium or format, as long as you give appropriate credit to the original author(s) and the source, provide a link to the Creative Commons license, and indicate if changes were made. The images or other third party material in this article are included in the article's Creative Commons license, unless indicated otherwise in a credit line to the material. If material is not included in the article's Creative Commons license and your intended use is not permitted by statutory regulation or exceeds the permitted use, you will need to obtain permission directly from the copyright holder. To view a copy of this license, visit http://creativecommons. org/licenses/by/4.0/.

(c) The Author(s) 2018 